\title{
Treatment Patterns and Clinical Characteristics of Patients with Advanced Basal Cell Carcinoma in the Community Oncology Setting*
}

\author{
Mark S. Walker ${ }^{1 \#}$, Lee S. Schwartzberg ${ }^{2}$, Diana M. Chen ${ }^{3}$, D. Devi Ramanan ${ }^{3}$, Arthur C. Houts ${ }^{1}$, \\ Carolina Reyes ${ }^{3}$
}

${ }^{1}$ ACORN Research, LLC, Memphis, USA; ${ }^{2}$ The West Clinic, Memphis, USA; ${ }^{3}$ Genentech, Inc., South San Francisco, USA.

Email: "mwalker@acorncro.com

Received May $15^{\text {th }}$, 2013; revised June $18^{\text {th }}$, 2013; accepted June $25^{\text {th }}, 2013$

Copyright (C) 2013 Mark S. Walker et al. This is an open access article distributed under the Creative Commons Attribution License, which permits unrestricted use, distribution, and reproduction in any medium, provided the original work is properly cited.

\begin{abstract}
Advanced basal cell carcinoma (aBCC) includes metastatic and locally advanced BCC that is inoperable (or with surgery contraindicated). We describe patient characteristics and treatment history for aBCC cases from community oncology. Nine cases of aBCC were found within the ACORN Data Warehouse, a community oncology database of $>180,000$ cancer patients. Data were summarized descriptively. Three illustrative case histories are presented. Patients were predominantly Caucasian (8/9), male (6/9), and over 60 (6/9). Four had metastatic disease; five had aBCC without metastasis. Five had a history of treatment for early stage BCC, including surgery (5/5), radiation (1/5), and none had chemotherapy. Those with history of early stage BCC had periods of apparent lack of follow-up and treatment. One had chemotherapy for aBCC (platinum based with radiation) and eight had radiation without chemotherapy. Patients had multiple comorbid serious medical conditions. Six were deceased, but only one was documented to have aBCC as cause of death. Advanced BCC is rare in community oncology settings. There appear to be gaps in the care and follow-up of patients with initial early stage BCC. More data and larger samples are needed from multi-specialty databases such as dermatology and head and neck surgery.
\end{abstract}

Keywords: Advanced Basal Cell Carcinoma; Community Oncology; Treatment Patterns; Observational Study; Long Term Follow Up

\section{Introduction}

Basal cell carcinoma (BCC) accounts for approximately $80 \%$ of nonmelanoma skin cancers [1]. Based on limited evidence from 2006, the incidence of basal cell and squamous cell skin cancer may be as high as 3.5 million cases annually [2]. The incidence is difficult to estimate because skin cancers are typically not tracked by cancer registries [2].

Basal cell carcinoma metastasizes only rarely, with estimates of about $0.1 \%$ of cases [3]. Median survival in

\footnotetext{
*Funding Source: The study reported in this paper was funded by Genentech, Inc., South San Francisco, CA, USA.

Conflict of Interest Statement: LSS is on the speakers' bureau for Genentech. DMC and CR are currently employed by Genentech, and DDR was previously employed by Genentech. MSW and ACH report that they have no conflicts to disclose.

Prior Presentation: Poster presentation at the $70^{\text {th }}$ Annual Meeting of the American Academy of Dermatology.

${ }^{\#}$ Corresponding author.
}

metastatic basal cell carcinoma is reported to be about 8 14 months [4-7]. The biological characteristics of BCCs that may predispose some of these cancers to behave aggressively or become metastatic are not well understood, [8] and recent investigations failed to identify immunohistochemical markers that distinguished metastatic and nonmetastatic BCCs [9]. Although a limited number of cases have been reported in the literature, [10] metastatic BCC is most often treated with combination chemotherapy; however, there have been no clinical trials evaluating chemotherapy [11,12]. Newer targeted therapies based on inhibition of the hedgehog pathway have shown promise in early trials $[13,14]$. On January 30,2012 , vismodegib was approved for the treatment of adults with metastatic basal cell carcinoma, or locally advanced BCC that has recurred following surgery or who are not candidates for surgery, and who are not candidates for radiation [15]. 
Nonmetastatic BCC may be treated with electrodessication and curettage [16], surgical excision, or with Mohs micrographic surgery $[17,18]$. Patients with lesions that are not surgically accessible, or who have recurrent lesions, may be treated with radiation therapy $[19,20]$. Patients with locally advanced or otherwise inoperable basal cell carcinoma may be viewed as higher risk, and candidates for chemotherapeutic intervention [21].

The incidence of metastatic BCC is very low. The rate of advanced or inoperable BCC is also rare but accurate incidence rates are not available. Most nonmelanoma skin cancers are treated outside medical oncology practices, a limitation of this analysis. As a result, patient samples available within typical community oncology practices are very small, and understanding of treatment patterns and outcomes in this population is correspondingly limited [22].

This study was a retrospective investigation to examine the patient characteristics, treatment patterns, and disease course of patients with advanced basal cell carcinoma (aBCC) treated in the community oncology setting.

\section{Patients and Methods}

\subsection{Patients}

Patients were eligible for this investigation if they were found during review to have had aBCC at any point from January 1, 2004 to November 1, 2010. Advanced BCC was defined as meeting either of two criterion based definitions: 1) locally advanced (i.e., American Joint Committee on Cancer [AJCC] Stage III) BCC [23] where per the medical record the patient was declared inoperable or where surgery was declared to be contraindicated, with receipt of radiation therapy unless radiation was contraindicated; or 2) metastatic BCC.

To be included, the patient had to be 18 years old or older and have received treatment at a community oncology practice affiliated with ACORN Research, LLC (Memphis, TN). Potentially eligible patients were identified through electronic search of patients with a diagnosis of BCC (ICD-9 173.xx) within the ACORN Data Warehouse, a repository containing records for 180,000 patients from 10 participating community practices in 10 states (CO, DE, GA, MD, MI, MS, MT, NC, OH, TN). Final eligibility was established through medical record review by experienced clinical research nurses who applied the aforementioned inclusion criteria defining aBCC case status.

\subsection{Study Design}

This study was a non-interventional study involving retrospective review of existing data collected as part of routine care. No direct patient contact occurred as part of this retrospective study.

The primary objective of this study was to describe the patients and treatment patterns observed from oncology medical records of aBCC patients treated in the community oncology setting. Given the low prevalence of aBCC, results were expected to be limited to a small number of patients. Reporting was therefore planned to include illustrative cases of three case types: 1) aBCC patient with history in the medical record of previous early stage disease prior to advanced disease; 2) aBCC patient without metastasis; 3) aBCC patient with metastasis. All study procedures were approved by IntegReview Institutional Review Board (Austin, TX).

\subsection{Study Measures}

Basic demographic characteristics were collected, including age at initial diagnosis of BCC, sex, and race. Disease characteristics included date and AJCC stage of initial BCC diagnosis, status as metastatic vs. nonmetastatic, location and size of lesions, and sites of metastasis among metastatic patients. Assessment of treatment patterns included history and dates of surgical treatments, radiation therapy, and chemotherapy prior to diagnosis of advanced disease and after diagnosis of aBCC. For chemotherapy treatment of aBCC, name of drug, dose and schedule were recorded. The specialty of providers was collected where available to infer whether patients received Mohs micrographic surgery, surgical excision, or radiation therapy. Comorbid conditions and performance status were also collected. The comorbid conditions that were documented included those assessed as part of the standard Charlson Comorbidity Index [24]. In addition, dates of each disease progression, date of death, and cause of death were assessed.

\subsection{Procedures}

Screening procedures used to identify possible cases included identification of cases with ICD-9 diagnosis codes for non-melanoma skin cancer (173.xx) and the use of words or phrases ("BCC," "basal cell”) indicative of basal cell carcinoma in searchable text within medical records. Screening required direct examination of each medical record, with electronic search within the record for text references to BCC, and review of the context of those references. Pathology records were also reviewed where available. The screening process ruled out cases of early stage BCC and references to personal or family history of BCC without any reference to the criteria that defined aBCC. These screening procedures resulted in identification of patients who met the aforementioned definition of aBCC used in this study. 


\subsection{Statistical Methods}

Statistical reporting was entirely descriptive, and included percentages, means, and standard deviations to describe the patient and disease-related characteristics.

\section{Results}

An initial screening list of over 1000 cases was created using ICD-9 codes and text references suggesting BCC. Following manual review of 1020 cases, nine patients with aBCC were identified within the ACORN Data Warehouse, and this represents the final study sample. Figure $\mathbf{1}$ presents a diagram showing reasons for screen failure. As shown, most cases were screen failures because of a personal history of BCC without evidence of locally advanced disease.

Table 1 shows demographic characteristics of the nine patients with aBCC. Average age at initial diagnosis of BCC was 61 years with a 2:1 ratio of males to females. Most patients were Caucasian, but race was not known for one patient. Text search of the record was used to identify indications of performance status impairment; however, formal performance status ratings were generally not available. None of the patients were impaired at the time of their aBCC diagnosis.

Table 2 shows disease and clinical characteristics for the nine patients with aBCC. Four of the nine patients were initially diagnosed with disease that was already locally advanced or metastatic, and had no history of prior treatment for BCC. The remaining five were diagnosed at an earlier stage, but specific stage was recorded in the medical records for only two of these patients (one each at AJCC stage I and II) [23]. The mean interval between the first record of BCC to diagnosis of aBCC in these patients was 6.9 years, with a range of less than one year (two patients) to more than 13 years (two patients).

Four patients were classified as aBCC due to metastatic disease, and the other five had locally advanced but nonmetastatic disease. Only two of the five locally advanced, nonmetastatic patients had a prior history of early stage disease. In contrast, three of the four metastatic patients were originally diagnosed and treated with early stage disease. Histopathological types were not available for one of the metastatic and one of the nonmetastatic aBCC cases. Otherwise, the most common types were infiltrating (3/9) and nodular (2/9).

Two-thirds of the patients had at least one significant comorbidity. Diabetes was the most prevalent, observed in three patients. This was followed in prevalence by chronic obstructive pulmonary disease, renal disease, and stomach ulcer with two patients each. Four patients had other cancers, including one bladder cancer, one colorectal cancer, and two patients with lung cancer. These

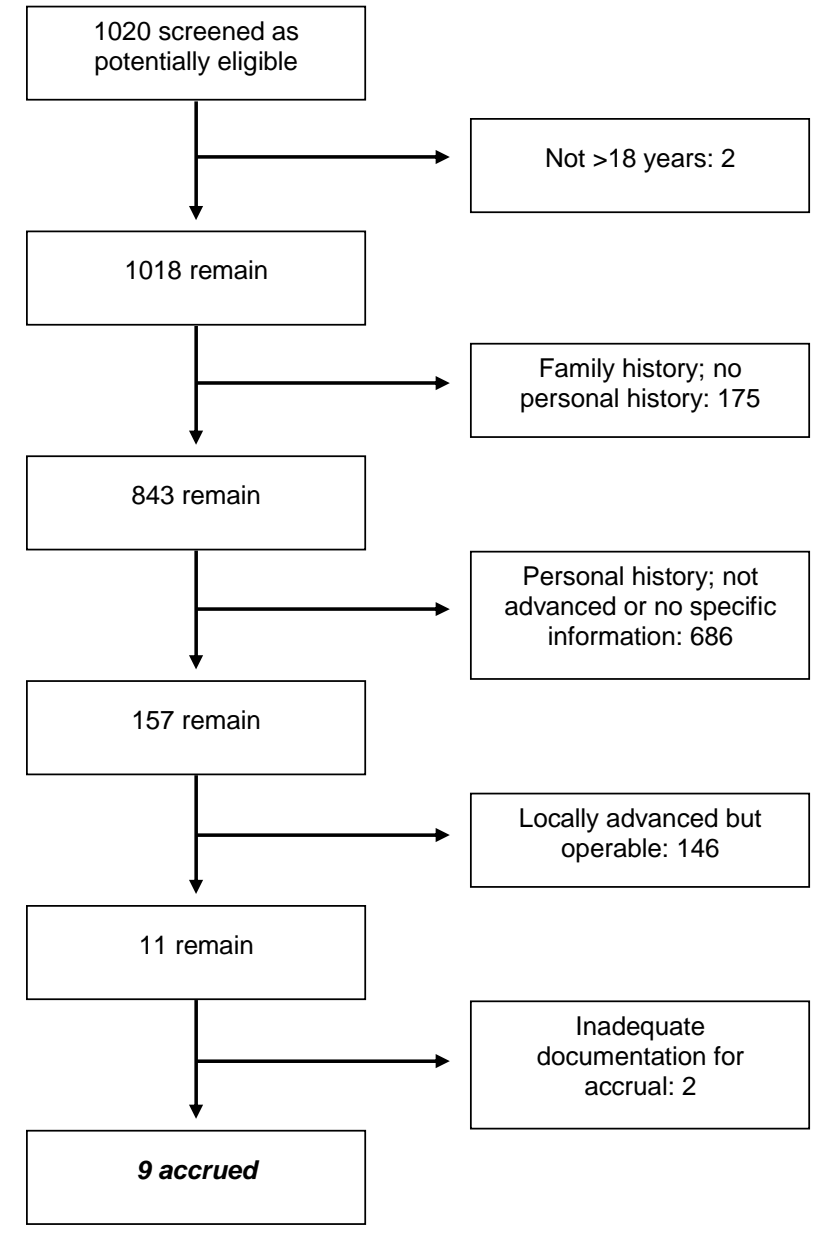

Figure 1. Basal cell carcinoma. Diagram of screening and accrual.

Table 1. Demographic characteristics of advanced basal cell carcinoma patients (aBCC).

\begin{tabular}{cc}
\hline Demographic characteristics & N (\%) \\
Sex & $61.10(13.33) ; 39-78$ \\
Male & $6(66.7 \%)$ \\
Female & $3(33.3 \%)$ \\
Race & \\
Caucasian & $8(88.9 \%)$ \\
Unknown/Not documented & $1(11.1 \%)$ \\
Performance status & \\
Impaired & \\
Not indicated as impaired & $9(100 \%)$ \\
\hline
\end{tabular}

*ECOG 2+ or text indication of impairment equivalent to ECOG 2+. 
Table 2. Disease and clinical characteristics of advanced basal cell carcinoma patients (aBCC).

\begin{tabular}{|c|c|}
\hline Disease and clinical characteristic & N (\%) \\
\hline \multicolumn{2}{|l|}{ Stage at initial presentation } \\
\hline Early stage BCC & $5(55.6 \%)$ \\
\hline Locally advanced or metastatic (aBCC) & $4(44.4 \%)$ \\
\hline \multicolumn{2}{|l|}{ aBCC Status and Histopathology Type } \\
\hline Metastatic & $4(44.4 \%)$ \\
\hline No subtype reported & 1 \\
\hline Infiltrating & 2 \\
\hline Poorly differentiated BCC & 1 \\
\hline Locally Advanced, not metastatic & $5(55.6 \%)$ \\
\hline No subtype reported & 1 \\
\hline Positive for BCC no other reported & 1 \\
\hline Nodular & 2 \\
\hline Infiltrating & 1 \\
\hline $\begin{array}{l}\text { Years from initial BCC to aBCC; } \\
\text { Mean (SD); Range* }\end{array}$ & 6.9 (6.7); $0.8-14.8$ \\
\hline \multicolumn{2}{|l|}{ Comorbidities within aBCC sample } \\
\hline Number of patients with at least 1 comorbidity & $6(66.7 \%)$ \\
\hline Chronic obstructive pulmonary disease & $2(22.2 \%)$ \\
\hline Congestive heart failure & $1(11.1 \%)$ \\
\hline Diabetes & $3(33.3 \%)$ \\
\hline Peripheral vascular disease & $1(11.1 \%)$ \\
\hline Renal disease & $2(22.2 \%)$ \\
\hline Stomach ulcer & $2(22.2 \%)$ \\
\hline
\end{tabular}

"Among five patients diagnosed initially with early stage BCC.

four patients included three who did not have a record of any other specific comorbidity. Taken together, the comorbid disease and other cancer burden reflect a patient sample with very serious health problems, apart from aBCC.

Table 3 shows the modalities of treatment for the subset of five patients who had records of early stage disease and for the entire sample of nine patients treated during the course of aBCC. Among the five patients who were treated during early stage disease, none received chemotherapy, one received radiation therapy, and all five had surgery. Documentation in the oncology medical record did not specify whether any of these were Mohs surgery. All five of these patients necessarily experienced progression to aBCC, since inclusion criteria required this. Only one of the nine patients with aBCC re-
Table 3. Treatment modalities during early stage disease and during advanced basal cell carcinoma.

\begin{tabular}{ccc}
\hline Type of treatment & $\begin{array}{c}\text { Early stage disease } \\
(\mathrm{N}=5)\end{array}$ & aBCC (N = 9) \\
\hline Chemotherapy, n (\%) & $0(0 \%)$ & $1(11.1 \%)$ \\
Radiation treatment, n (\%) & $1(20.0 \%)$ & $9(100 \%)$ \\
Surgical treatment, n (\%) & $5(100 \%)$ & -- \\
\hline
\end{tabular}

Note: Only 5 patients had treatment of Early Stage Disease and all progressed to advanced basal cell carcinoma (aBCC).

ceived chemotherapy. That patient was treated with chemoradiation, including cisplatin and paclitaxel weekly for eight weeks, with a single dose of carboplatin at the end. After 21 month follow-up, this patient did not have disease progression following the chemoradiation treatment. Among the eight patients who did not have chemotherapy, four developed disease progression. As shown in Table 3, all nine aBCC patients received radiation therapy in the aBCC setting, including the afore-mentioned chemoradiation treatment. Some patients received sequential radiation therapy to different lesions. Two patients received radiation therapy that was described as palliative, and one of these received only palliative radiation therapy in the aBCC setting.

Illustrated in Table $\mathbf{4}$ is a mortality table for all nine patients. Death status was determined from the clinical record and with Social Security Death Index. Three of the nine were still living at the close of the study. Among the six who were deceased, only one had a cause of death likely attributable to BCC. Two patients died due to lung cancer or likely due to lung cancer. Three patients had cause of death that was unknown or unstated in the available records.

\section{Case Examples}

History of Previous Early Stage Disease Prior to aBCC (ID \#8). This patient was a white male diagnosed with a right temporal BCC in 2008 at the age of 63 . The patient was reported in the medical record to have had chronic obstructive pulmonary disease and a history of renal disease. His ECOG performance status was reported as 0 . The size and AJCC staging of the initial BCC lesion was not reported in the available medical record. This lesion was resected, and the patient received radiation therapy. The original pathology was not available, and no histological information was reported in the record. The patient then presented 10 months after initial presentation with swelling in his right neck. A biopsy was performed of the parotid and jugular lymph nodes and was consistent with metastatic BCC, infiltrating type. The parotid lymph node metastasis was reported as $2 \mathrm{~cm}$. The size of 
the jugular lymph lesion was not reported. The patient had gross resection of the parotid lymph node and two jugular chain nodes. He also received chemoradiation with chemotherapy consisting of cisplatin/paclitaxel for an eight week period beginning at month 11 . He received carboplatin/paclitaxel for the last treatment. A computerized tomographic (CT) scan was performed at month 14, and compared with a CT scan done at month 9, prior to the chemoradiation. The scan findings were "unremarkable" and "normal". There was no evidence of pathologically enlarged adenopathy in the neck. The scan report did not reference any evidence of disease. A followup progress note cited "no evidence of recurrence ... [the patient] having had gross resection of the disease in the parotid region and having completed definitive chemoradiation to the parotid lymph node region and neck." The patient was last seen at month 34 and was stable. There was no record of the patient's death.

Advanced Basal Cell Carcinoma without Metastasis (ID \#1). The patient was a white female, first diagnosed with BCC in 1990 at the age of 64. Her medical oncology chart begins in 2001, when she was diagnosed with early stage colon cancer. The patient had a history of congestive heart failure and diabetes. Although the patient's oncology chart begins in 2001, there was no mention of her history of BCC at this point. Instead, the first note related to BCC was a pathology report dated in 2005. This pathology report mentioned BCC recurrence of the right scalp, extending to margins of resection. There were no additional histopathology findings in the report. The medical oncologist's notes did not address the BCC until about month 6 after the pathology note, when the medical record stated that the patient had a 15-year history of right scalp BCC with five resections, according to the patient, and recurrences. The dates of the resections were not reported, and the specialties of the providers who performed the resections were also not reported. This BCC, which had grown to $12 \times 18 \mathrm{~cm}$, was considered locally advanced BCC. The medical oncologist stated in this note that the lesion was now too large to be resected with local anesthesia, and that the patient was unable to have general anesthesia due to her heart disease. She was a candidate for radiotherapy, which she received to her right scalp for five weeks beginning month 7 . The patient did not receive any chemotherapy for her BCC. At the patient's follow-up visit at month 9, about one month following the completion of radiotherapy, the medical oncologist stated that the lesion was healing nicely and instructed her to follow-up with her dermatologist. The patient continued to be followed for her colon cancer without any further mention of the BCC. The patient was last seen in 2008, at month 33, and this note addressed her colon cancer only. There was no record of death in the patient's oncology chart, but she died at month 53 according to the Social Security Death Index, at about age 83. No information regarding cause of death was available.

Advanced Basal Cell Carcinoma with Metastasis (ID \#9). This was a male patient, race unspecified, who was diagnosed with metastatic BCC of the left ear extending to the left temporal bone in 2009 at the age of 75 . The patient was reported in the medical record to have chronic obstructive pulmonary disease, a history of renal disease, and ulcer disease. The patient's history also included a possible cardiovascular accident. No histopathology was available. The patient's initial BCC was AJCC stage IV according to the chart record, and the patient received radiation therapy, ending at about month 3 after initial diagnosis. The duration of the radiation therapy was not reported. No chemotherapy was given. The patient did well until about month 6 when a follow-up magnetic resonance imaging (MRI) study showed metastases of the C4 and $\mathrm{T} 1$ vertebral bodies. A biopsy of the T1 lesion revealed metastatic, poorly differentiated BCC. No treatment was given at this time. A bone scan at month 8 revealed metastatic tumor in three portions of the skull, the cervical spine and upper thoracic spine. The patient then received three weeks of radiation therapy to his spine. The medical oncologist considered chemotherapy but decided against this due to the patient's lack of symptoms. The patient had a follow-up MRI at month 12 that revealed a tumor in the lateral wall of the left orbit. He received radiation therapy to this area, ending at month 13 . The last note in the chart was dated at month 14 when the patient was instructed to have a follow-up MRI in two months. The medical oncologist discussed the possibility of chemotherapy if the patient developed systemic disease not amenable to palliative radiation therapy. There were no other notes in the chart. The chart did not document a cause of death, but the patient was reported by Social Security Death Index to have died at about month 17.

\section{Discussion}

Advanced basal cell carcinoma is rare and information about its treatment in the community setting is limited. We were able to identify only nine such cases in a community oncology data warehouse of 180,000 patients. Six of those nine were deceased. Precise causes of death among the six deceased patients were not well documented; in only one case of the six was there any reference to BCC as the cause of death. Two-thirds of the patients were over 60 years old and had a variety of other medical conditions, including other cancers, indicating generally poor health. Selection of cases from medical oncology 
practices may have biased the sample toward patients with other cancers, however.

Five of the nine patients had a history of BCC prior to developing aBCC, and the oncology medical records often lacked detail regarding the history of treatment of the early stage disease. AJCC staging for BCC is not routinely used in other specialties such as dermatology. There is a lack of universal staging criteria and accordingly this contributes to the challenges in identifying aBCC cases. Information regarding location and size of lesions at initial presentation was incomplete and reasons for lack of follow-up were often unclear. The extent to which the lack of information in the oncology record was due to the patient or physician is also unclear. For example, in the case study illustration of aBCC that was not metastatic (ID \#1), the patient had a long history of BCC prior to aBCC. Even though the patient reported a history of five previous BCC resections to the scalp, it appears that information on the recurrent BCC on the scalp had not been captured over the course of a four-year period when he was being followed in medical oncology for colon cancer. In another case (ID \#4 in Table 4), a patient had a 15year history of BCC that included two resections, one due to recurrence, but at the time the patient was seen for advanced disease, the patient had neglected or was unaware of a second recurrence for over two years. In yet another case (ID \#3), a patient observed a lesion on his face growing to a size of $3 \times 5 \mathrm{~cm}$ over a period of 8 years, yet the patient sought treatment only after the lesion began to itch.

It is unclear why patients may wait long periods of time before seeking the attention of medical profession- als, or why when care is sought that recurrent disease is missed. It is possible that given the multiple medical conditions of these patients, that patients and physicians do not place a high degree of attention to skin cancer. Efforts to educate the public about the dangers of skin cancer have been fairly recent, and it is possible that there are cohort effects in that those who develop aBCC have never received such education. The treatment course in aBCC needs to be examined in larger data sets that include multiple specialties that treat BCC to better understand this patient population and the evolution of their BCC care over the long term.

This study was limited by the small number of patients identified. There simply may be a small proportion of all aBCC patients who are treated in community oncology practices as opposed to other settings. An additional limitation was that medical records available were limited to those within the medical oncology practice. As a result, detailed records regarding earlier treatment, including surgery, were lacking. Further, because patients were selected from among those seen in medical oncology practices, there may have been a bias toward selection of cases with other cancers. Although such a selection bias may have been present, it is also important to note that our sample was demographically similar to another larger community oncology sample in being predominantly male, over 60 years old, and with significant comorbidities [22].

Although we identified these cases from a repository of 180,000 patients, there is clearly a need to have richer sources of information about aBCC. This may be achievable with larger samples where both claims data and

Table 4. Mortality table for nine aBCC patients.

\begin{tabular}{|c|c|c|c|c|c|c|c|}
\hline ID & aBCC status & Age at initial $\mathrm{dx}$ & Sex & Deceased & Cause of death & Other disease burden & $\begin{array}{l}\text { Years to end of } \\
\text { observation }\end{array}$ \\
\hline 1 & LA & 64 & $\mathrm{~F}$ & $\mathrm{Y}$ & Unknown & Colon cancer, CHF, diabetes & 4.48 \\
\hline 2 & LA & 71 & $\mathrm{M}$ & $\mathrm{Y}$ & Lung cancer & Lung cancer & 3.22 \\
\hline 3 & LA & 61 & $\mathrm{M}$ & $\mathrm{Y}$ & Not stated & Bladder cancer & 0.97 \\
\hline 5 & LA & 39 & $\mathrm{~F}$ & $\mathrm{~N}$ & -- & Diabetes, ulcer disease & 0.5 \\
\hline 6 & Met & 47 & M & $\mathrm{Y}$ & Possibly lung cancer & Lung cancer, metastatic & 7.5 \\
\hline 7 & Met & 78 & $\mathrm{M}$ & $\mathrm{N}$ & -- & $\begin{array}{l}\text { Diabetes, peripheral vascular } \\
\text { disease, history of CABG }\end{array}$ & 2.96 \\
\hline 9 & Met & 75 & $\mathrm{M}$ & $\mathrm{Y}$ & Unknown & $\begin{array}{l}\text { COPD, history of renal disease, } \\
\text { ulcer disease }\end{array}$ & 1.46 \\
\hline
\end{tabular}

Note. Three patients remained alive at the time of record inspection. Years to end of observation = total time under observation from aBCC diagnosis until death or censoring. Abbreviations used: LA (Locally advanced), Met (Metastatic), CHF (congestive heart failure), CABG (coronary artery bypass graft), COPD (chronic obstructive pulmonary disease), Dx (diagnosis), N (No), Y (Yes). 
detailed medical histories can be combined to identify cases from multiple treatment settings.

As new treatments become available in the emerging era of targeted therapies, it is important to understand the history and patterns of treatment in such poorly described cancers as advanced BCC so that both patients and providers can fully appreciate the impact of future treatments.

\section{Acknowledgements}

ACORN wishes to express its appreciation for the participation of the practices and patients whose data were used in this study. The authors also acknowledge the contribution of Jiandong Kerr, MS, ACORN Research, who performed the statistical analyses for this paper. Mr. Kerr has no pertinent conflicts of interest to disclose.

\section{REFERENCES}

[1] A. I. Rubin, E. H. Chen and D. Ratner, "Basal-Cell Carcinoma,” New English Journal of Medicine, Vol. 353, No. 21, 2005, pp. 2262-2269. doi:10.1056/NEJMra044151

[2] American Cancer Society, "Cancer Facts and Figures 2011,” American Cancer Society, 2011. http://www.cancer.org/acs/groups/content/@epidemiolog ysurveilance/documents/document/acspc-029771.pdf

[3] Bader RS, “Basal Cell Carcinoma. eMedicine,” 2012. http://emedicine.medscape.com/article/276624-overview

[4] J. S. Lo, S. N. Snow, G. T. Reizner, F. E. Mohs, P. O. Larson and G. J. Hruza, "Metastatic Basal Cell Carcinoma: Report of Twelve Cases with a Review of the Literature," Journal of the American Academy of Dermatology, Vol. 24, No. 5, 1991, pp. 715-719. doi:10.1016/0190-9622(91)70108-E

[5] H. von Domarus and P. J. Stevens, "Metastatic Basal Cell Carcinoma. Report of Five Cases and Review of 170 Cases in the Literature," Journal of the American Academy of Dermatology, Vol. 10, No. 6, 1984, pp. 1043-1060.

[6] R. L. Raszewski and B. Guyuron, "Long-Term Survival Following Nodal Metastases from Basal Cell Carcinoma,” Annals of Plastic Surgery, Vol. 24, No. 2, 1990, pp. 170175. doi:10.1097/00000637-199002000-00012

[7] A. Wadhera, M. Fazio, G. Bricca and O. Stanton, "Metastatic Basal Cell Carcinoma: A Case Report and Literature Review. How Accurate Is Our Incidence Data?” Dermatology online Journal, Vol. 12, No. 5, 2006, p. 7

[8] H. W. Walling, S. W. Fosko, P. A. Geraminejad, D. C. Whitaker and C. J. Arpey, “Aggressive Basal Cell Carcinoma: Presentation, Pathogenesis, and Management," Cancer Metastasis Reviews, Vol. 23, No. 3-4, 2004, pp. 389-402. doi:10.1023/B:CANC.0000031775.04618.30

[9] D. N. Ionescu, M. Arida and D. M. Jukic, "Metastatic Basal Cell Carcinoma: Four Case Reports, Review of Literature, and Immunohistochemical Evaluation,” Archives of Pathology \& Laboratory Medicine, Vol. 130, No. 1,
2006, pp. 45-51.

[10] P. T. Ting, R. Kasper and J. P. Arlette, "Metastatic Basal Cell Carcinoma: Report of Two Cases and Literature Review," Journal of Cutaneous Medicine and Surgery, Vol. 9, No. 1, 2005, pp. 10-15. doi:10.1007/s10227-005-0027-1

[11] B. A Carneiro, W. G Watkin, U. K. Mehta and B. E. Brockstein, "Metastatic Basal Cell Carcinoma: Complete Response to Chemotherapy and Associated Pure Red Cell Aplasia,” Cancer Investigation, Vol. 24, No. 4, 2006, pp. 396-400. doi:10.1080/07357900600705474

[12] J. D. Khandekar, "Complete Response of Metastatic Basal Cell Carcinoma to Cisplatin Chemotherapy: A Report on Two Patients," Archives of Dermatology, Vol. 126, No. 12, 1990, p. 1660. doi:10.1001/archderm.1990.01670360128037

[13] J. A. Low and F. J. de Sauvage, "Clinical Experience with Hedgehog Pathway Inhibitors,” Journal of Clinical Oncology: Official Journal of the American Society of Clinical Oncology, Vol. 28, No. 36, 2010, pp. 5321-5326. doi:10.1200/JCO.2010.27.9943

[14] D. D. Von Hoff, P. M. LoRusso, C. M. Rudin, J. C. Reddy, R. L. Yauch, R. Tibes, G. J. Weiss, M. J. Borad, C. L Hann, J. R. Brahmer, H. M. Mackey, B. L. Lum, W. C. Darbonne, J. C. Marsters Jr., F. J. de Sauvage and J. A. Low, "Inhibition of the Hedgehog Pathway in Advanced Basal-Cell Carcinoma,” New English Journal of Medicine, Vol. 361, No. 12, 2009, pp. 1164-1172. doi:10.1056/NEJMoa0905360

[15] Genentech, “Drug Label for Vismodegib,” Genentech, 2012.

http://www.gene.com/gene/products/information/erivedge /pdf/erivedge_prescribing.pdf?cid=erv_we_F001110_P00 0517\&c=MBEVUA1012\&utm_source $=\& u t m \_$medium $=$c pc\&utm_term=advanced\%20basal\%20cell\%20carcinoma \&utm_campaign=2012\%20Erivedge\%20Condition\%20(J an12)\&gclid=CP2Si-rM164CFQ1U7AodiEo9bg

[16] National Cancer Institute, "Basal Cell Carcinoma of the Skin," 2008.

http://www.cancer.gov/cancertopics/pdq/treatment/skin/H ealthProfessional/page5

[17] R. Malhotra, S. C. Huilgol, N. T. Huynh and D. Selva, "The Australian Mohs Database, Part II: Periocular Basal Cell Carcinoma Outcome at 5-Year Follow-Up," OphthaImology, Vol. 111, No. 4, 2004, pp. 631-636. doi:10.1016/j.ophtha.2003.11.004

[18] R. M. Thomas and R. A., "Amonette Mohs Micrographic Surgery," American Family Physician, Vol. 37, No. 3, 1988, pp. 135-142.

[19] M. Caccialanza, R. Piccinno, S. Percivalle and M. Rozza, "Radiotherapy of Carcinomas of the Skin Overlying the Cartilage of the Nose: Our Experience in 671 Lesions," Journal of the European Academy of Dermatology and Venereology, Vol. 23, No. 9, 2009, pp. 1044-1049. doi:10.1111/j.1468-3083.2009.03247.x

[20] R. D. Lovett, C. A. Perez, S. J. Shapiro and D. M. Garcia, "External Irradiation of Epithelial Skin Cancer," International Journal of Radiation Oncology Biology Physics, 
Vol. 19, No. 2, 1990, pp. 235-242.

doi:10.1016/0360-3016(90)90529-S

[21] K. Moeholt, P. Pfeiffer, H. Aagaard and O. Hansen, “Local Recurrence of Basal Cell Carcinoma and Cisplatinum Containing Chemotherapy,” Acta Oncology, Vol. 36, No. 1, 1997, p. 87. doi:10.3109/02841869709100742

[22] A. D. Joshi, M. T. Forsyth, D. C. Fryefield, W. Lopez, D. M. Chen and D. D. Ramanan, "Demographic and Treatment Characteristics for Advanced Basal Cell Carcinoma (aBCC) in an Oncology Outpatient Setting," ASCO Meeting Abstracts, Vol. 29, No. S15, 2011, Article ID: e19001.
[23] S. B. Edge and American Joint Committee on Cancer, "Cutaneous Squamous Cell Carcinoma and Other Cutaneous Carcinomas,” In: S. B. Edge, D. R. Byrd, C. C. Compton, et al., Eds., AJCC Cancer Staging Manual, 7th Edition,. Springer, New York, 2010, pp. 301-314.

[24] M. E. Charlson, P. Pompei, K. L. Ales and C. R. MacKenzie, "A New Method of Classifying Prognostic Comorbidity in Longitudinal Studies: Development and Validation,” Journal of Chronic Diseases, Vol. 40, No. 5, 1987, pp. 373-383. doi:10.1016/0021-9681(87)90171-8 